\title{
INVOCACIONES DUPLICADAS A LOS DIOSES MANES EN INSCRIPCIONES ROMANAS DE HiSPANIA
}

\section{Roman Inscriptions from Hispania with the Invocation to the Manes Gods Repeated}

\author{
JUAN MANUEL ABASCAL PALAZÓN
}

Dpto. de Prehistoria, Arqueología, Hª Antigua, Filología Griega y Filología Latina. Universitat d’Alacant. juan.abascal@ua.es

\section{RESUMEN:}

Presentamos en estas páginas ocho testimonios de inscripciones romanas que muestran la repetición de la fórmula $\mathrm{D}$ (is) $\mathrm{M}$ (anibus) $\mathrm{s}$ (acrum) $o \mathrm{D}$ (is) $\mathrm{M}$ (anibus). Todos los textos pueden ser datados entre los ss. II y III. La distribución geográfica de estos textos demuestra que se trata de testimonios aislados y que no guardan entre ellos ninguna relación.

Palabras clave: epigrafía romana, formularios epigráficos, monumentos funerarios, Baetica, Hispania Citerior, estelas.

\section{Abstract:}

This article presents eight Roman inscriptions found in Hispania with reiteration of epigraphic formula $\mathrm{D}$ (is) $\mathrm{M}(\mathrm{anibus}) \mathrm{s}($ acrum) or $\mathrm{D}$ (is) $\mathrm{M}$ (anibus). All these testimonies can be dated between the II and III centuries A.D. The geographical distribution shows isolated samples without connection.

Key words: Roman epigraphy, epigraphic formula, funerary monuments, Baetica, Hispania Citerior, stelae.

Copyright: 12017 Juan Manuel Abascal. This is an open access paper distributed under the terms of the Creative Commons License, (CC BY-NC-SA 3.0), which permits unrestricted use, distribuof the Creative , and reproduction in any medium, provided the original author and source are credited.
tion 
Entre las inscripciones funerarias romanas de la península ${ }^{1}$ Ibérica se conservan algunos textos que presentan, bien al inicio, o jalonando el epígrafe por arriba y por abajo, invocaciones duplicadas implorando la protección de los dioses Manes. Bien es cierto que el número de testimonios de esta práctica es reducido, pero la ostentación con que en ocasiones se hace es suficiente para considerar que algunos testimonios no responden a un descuido del grabador sino a una repetición consciente.

Sin perjuicio de que pueda existir algún otro testimonio, en las páginas que siguen presentaremos los ejemplos que hemos podido recoger hasta el momento. Hemos excluido de esta relación una estela de Los Corrales (Sevilla, conventus Astigitanus) que señala el lugar de enterramiento de Grapta (Pastor y Rodríguez Martín 1999-2000: 232-233), en donde la pretendida duplicidad de la invocación de la fórmula D. M. s. (HEp 9, 1999, 505) no es demasiado evidente; aunque en sus comentarios a la edición original, A. Canto afirma que por debajo de la raya que termina el campo epigráfico se ha repetido otra vez la fórmula, más completa, DMS (HEp 9, cit.), no es fácil distinguir la presencia de esas letras.

\section{TESTIMONIOS DE INVOCACIONES DUPLICADAS}

\section{FUENTE DE PIEDRA (MÁLAGA) (CONVENTUS ASTIGITANUS, BAETICA)}

Altar funerario de caliza dedicado a la memoria de Flavius Sextio; fue descubierto en 1949 en el paraje de La Vicaría, en término de Fuente de Piedra (Málaga) y se conserva en el Museo de Málaga.

En el coronamiento, escrita en la parte frontal de los pulvinos y del fastigium, se lee la invocación $D(i s)$ M(anibus) [s(acrum)]. El texto grabado luego en la cartela comienza con la misma fórmula, lo que produce una duplicación de la invocación (fig. 1). Las dos primeras líneas del texto, en la transcripción editada por Atencia dicen:

$D$ (is) M(anibus) [s(acrum)]

$D($ is) M(anibus) s(acrum)

Bib.: Atencia 1971, 40 n. ${ }^{\circ}$ 18; Serrano y Atencia 1981: 53, n. ${ }^{\circ} 54$ (AE 1981, 507); Gozalbes y Muñoz 1986: 23 (HEp 1, 1989, 474). De ellos, Atencia, CIL $\mathrm{II}^{2} / 5,833$.
El formulario y el tipo de letra permiten fechar este monumento en la primera mitad del s. III.

\section{MENGÍBAR (JAÉN) (CONVENTUS CORDUBENSIS, BAETICA)}

Estela de arenisca coronada por dos pulvinos y frontón, como si se tratara de un altar, y rota por abajo. El texto está recuadrado por una incisión. Inicialmente se consideró procedente de la zona de Úbeda (Pastor y Stylow 1996: 288) y estuvo en una colección particular de esta localidad; luego pasó al mercado de antigüedades y reapareció en una colección privada de Valencia (Corell y Gómez 2002/03: 239), desde donde ingresó en el Servicio de Investigación Prehistórica (SIP) de esta localidad, en donde hoy se conserva.

Esta estela funeraria de Vibia Lavinia presenta escrita en el frontón superior la fórmula $D(i s)$ M(anibus) s(acrum). Sin embargo, en la última línea, hoy parcialmente perdida y por eso no reproducida aquí, se conserva la primera letra y el trazo izquierdo de la segunda, de manera que se puede volver a leer allí $D$ (is) M(anibus) [s(acrum)], como defendieron los primeros editores.

Bib.: Pastor y Stylow 1996: 288-290 y 292 n. ${ }^{\circ} 5$, con foto ( $A E$ 1996, 923; HEp 7, 1997, 372); Corell y Gómez 2002/03: 239 y 242-245 n. ${ }^{\circ}$ 2, con foto (HEp 12, 2002, 303), que rechazan la lectura $D$ (is) M(anibus) [s(acrum)] de la última línea.

\section{LUGO (CONVENTUS LUCENSIS, HISPANIA CITERIOR)}

Estela de piedra caliza, descubierta en 1936 en la plaza de Santo Domingo en Lugo, al hacer los cimientos del mercado. Se conserva en el Museo Provincial de Lugo, donde pudimos describirla y fotografiarla el 24 de junio de 2015.

El texto contiene la dedicación hecha por un padre para sus dos hijos, uno de los cuales se llamaba Paternus, que fueron asesinados en el mes de abril de un año desconocido. Al comienzo de la estela, de forma nítida, aparece repetida la invocación a los dioses Manes (fig. 2). El monumento ha sido editado en diferentes ocasiones, por lo que evitaremos aquí repetir el texto. Las dos primeras líneas dicen:

$D($ is) M(anibus) s(acrum) $D($ is) M(anibus) s(acrum) 


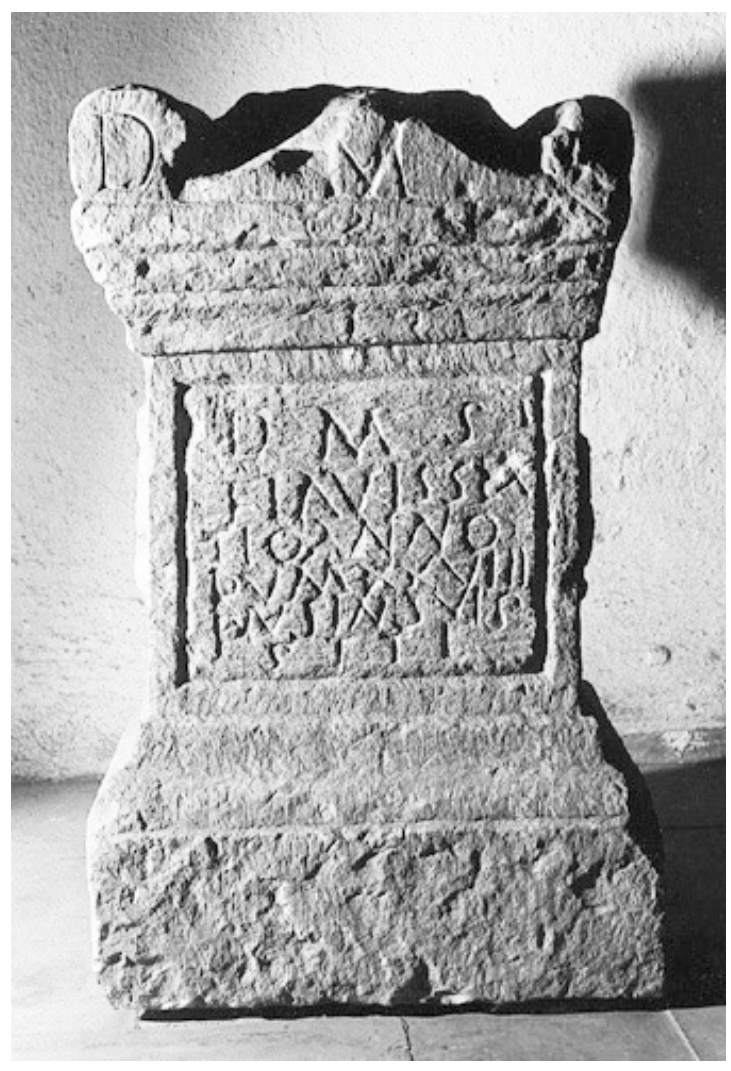

Fig. 1: Altar de Fuente de Piedra, según Atencia en CIL II.

Bib.: Vázquez Seijas 1948-1949: 186 n. ${ }^{\circ} 2$ (HAE 315; ILER 6443); D’Ors y Vázquez Seijas 1949: 205-207; Vázquez Saco y Vázquez Seijas 1954: 71-72 n. ${ }^{\circ} 43$ (HAE 1722); Rodríguez Lage 1974: 27-28 n. 35; Arias et al. 1979: 66-67 n. ${ }^{\circ}$ 43; Gómez Vila 2009: 191 n. ${ }^{\circ}$ 91; Rodríguez Colmenero 2011: 127 y fig. 62.

Las características formales del monumento, el formulario y el tipo de letra permiten fecharlo a finales del s. II o comienzos del III.

La duplicidad de la invocación a los dioses Manes sólo puede entenderse por ser dos los difuntos. El esquema de la composición del texto, mutatis mutandis, es muy similar al de una estela de mármol procedente de Hippo Regius (Hipona, Africa proconsularis. CIL VIII, 17437; Gsell 1922: n. ${ }^{\circ}$ 51), en cuya cabecera se repite la fórmula $D$. M. s. de manera muy similar a como se encuentra en la estela de Lugo. En la península Ibérica, el paralelo directo de esta repetición inicial de la fórmula en una estela es el monumento de El Salobral (infra n. ${ }^{\circ}$ 7).

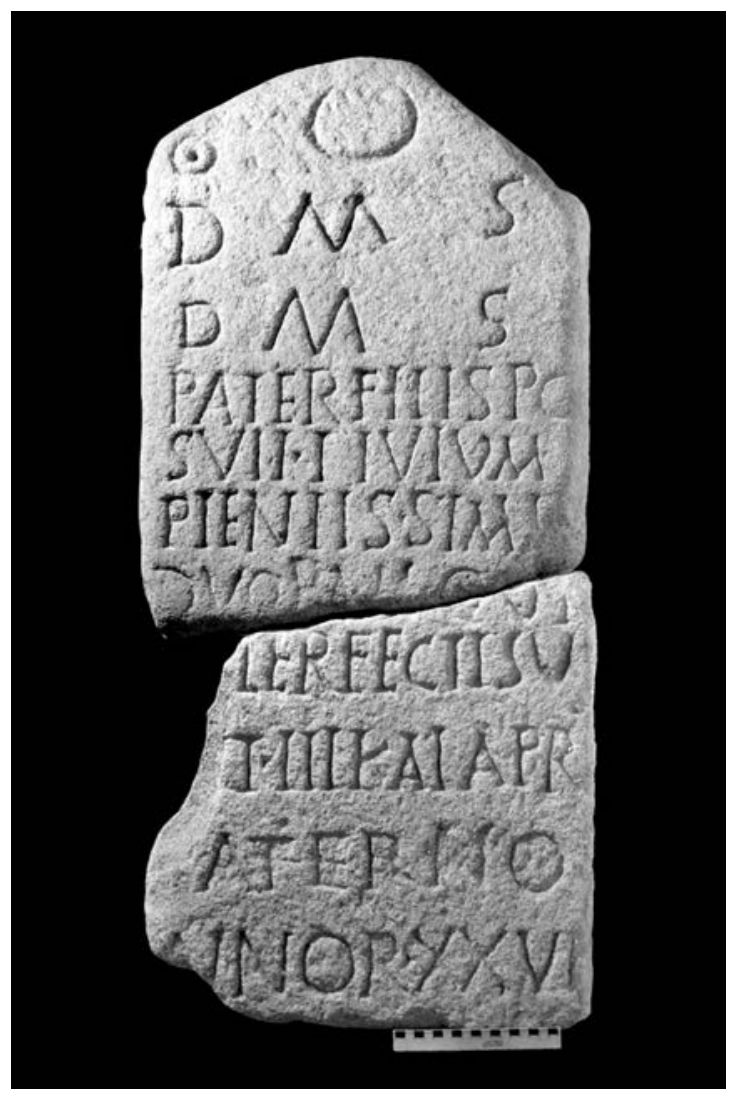

Fig. 2: Estela de Lugo. Foto: J. M. Abascal.

\section{SANTIBÁÑEZ DE VIDRIALES (ZAMORA) (CONVENTUS ASTURUM, HISPANIA CITERIOR)}

Estela de mármol blanco descubierta antes de 1923 en el paraje de "Ciudadeja" (Rosinos y Santibáñez de Vidriales, Zamora). Se encuentra depositada desde hace pocos años en el aula-museo del Ayuntamiento de Santibáñez de Vidriales, en donde la describimos y fotografiamos el 22 de junio de 2015.

En una cartela ligeramente rehundida se encuentra grabado el texto funerario, que comienza con la invocación $D$ (is) M(anibus) (fig. 3). Sin embargo, en el extremo inferior de la pieza, sin contexto y de manera completamente aislada, se volvió a grabar la fórmula $D(i s)$ $M$ (anibus) s(acrum) (fig. 4); en esta última línea se encuentra incluso repetida la letra inicial $D$. El texto completo de este monumento, que hemos estudiado recientemente en detalle (Abascal 2016: 347-351, con fotos y dibujos), dice: 


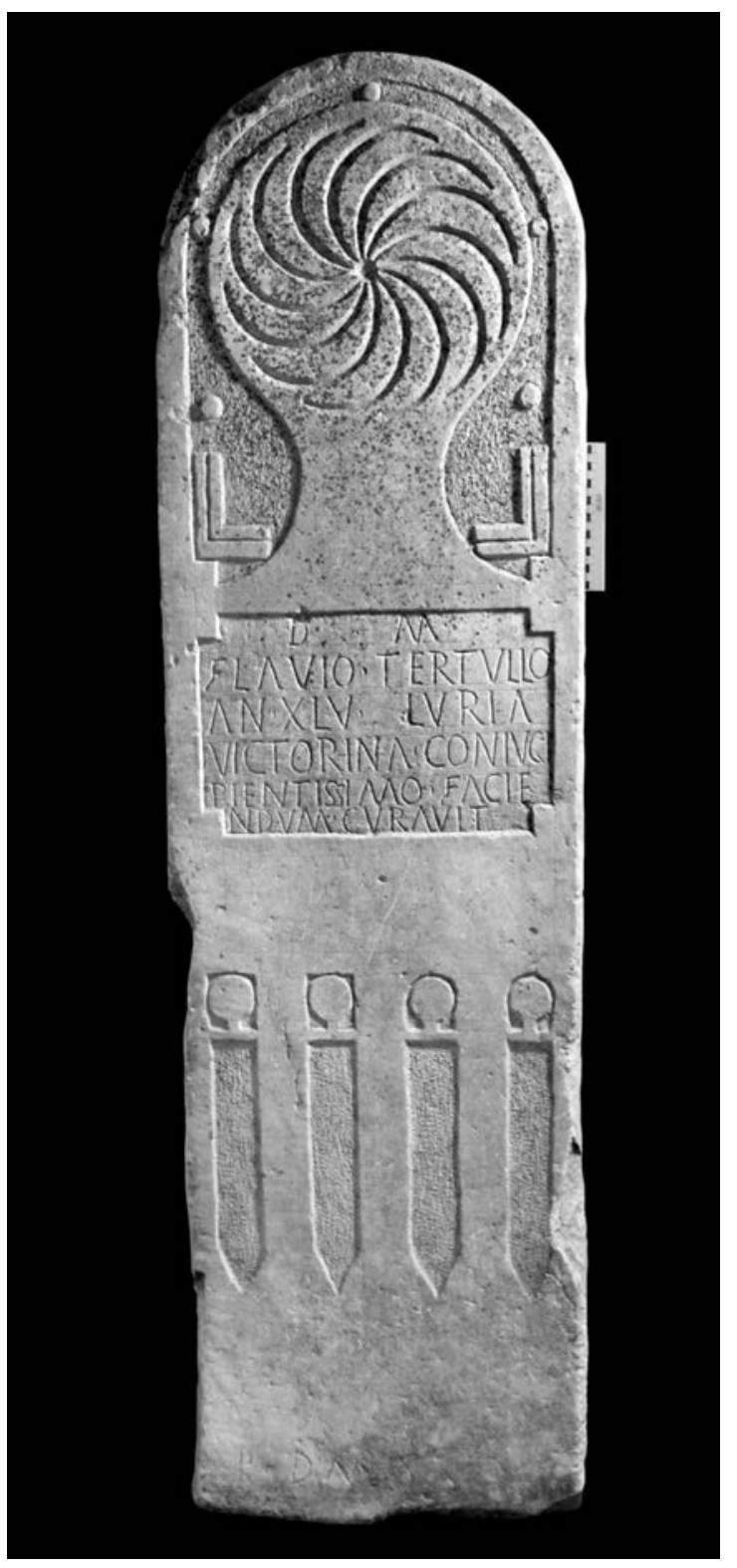

Fig. 3: Estela de Flavius Tertullus. Foto: J. M. Abascal.

$D$ (is) M(anibus)

Flavio $\cdot$ Tertullo

an(norum $) \cdot X L V \circ$ Luria

Victorina $\cdot$ coniugi

5 pientissimo $\cdot$ facie-

ndum $\cdot$ curavit

$D(--) \quad D($ is $)$ M(anibus) s(acrum) (!)

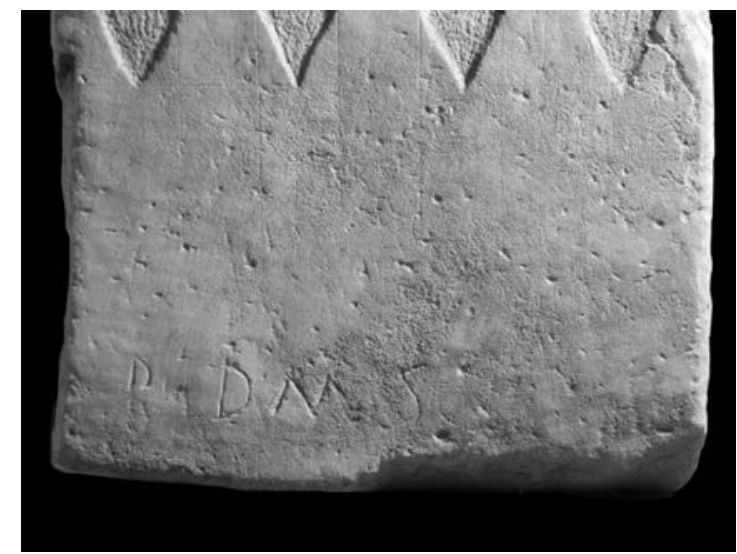

Fig. 4: Extremo inferior de la estela de Flavius Tertullus, con la segunda invocación. Foto: J. M. Abascal.

Bib.: Blázquez 1923: 333; Morán 1937, 142 Abb. 1; Id., El Correo de Zamora, 30 de junio de 1940; Martín Valls 1975, 15-16 y 18, Lám. IV.2 y V.1 (AE 1976, 290a); Bragado 1990, n. ${ }^{\circ}$ 98; Hernández 1999: 90-91 n. ${ }^{\circ} 69$ y 222 Lám. XVI; Alonso y Crespo 2000: 84-85 n. ${ }^{\circ}$ 181; Abascal 2016: 347-351. - Cf. Abásolo y García Rozas 1990: 545-546, 548, 554 n. o 88, 555 con Fig. 1, 559 Lám. III; Lión 1990: 564, 569 Lám. V.

El formulario y la estructura del texto sugiere una datación en la segunda mitad o finales del s. II.

La presencia al comienzo y al final del texto de la invocación $D$. $M$., esta vez sin $s$ (acrum), vuelve a encontrarse en una inscripción funeraria de Lovech (Bulgaria. Moesia inferior) que presenta una datación consular del año 233 (CIL III 6141). Por circunstancias distintas, la situación se repite en sendas estelas de Mengíbar y Castellar de Santisteban (supra n..$^{\circ}$ e infra n..$^{\circ}$ ).

\section{VALENCIA DE DON JUAN (LEÓN) (CONVENTUS ASTURUM, HISPANIA CITERIOR)}

Epitafio métrico grabado en una placa de caliza marmórea que contiene la dedicación hecha por Fortunata y Heliodorus para su hijo Pomponius. El monumento fue descubierto en 1950 en el Cerro de la Muela, en término de Valencia de Don Juan, y se conserva en un domicilio particular de esta localidad.

Según señaló Sebastián Mariner (1955: 239-243) y ha recalcado tras su análisis directo Javier del Hoyo (2001: 31), al comienzo de la segunda línea del texto, allá donde la editio princeps indicaba simplemente las letras 


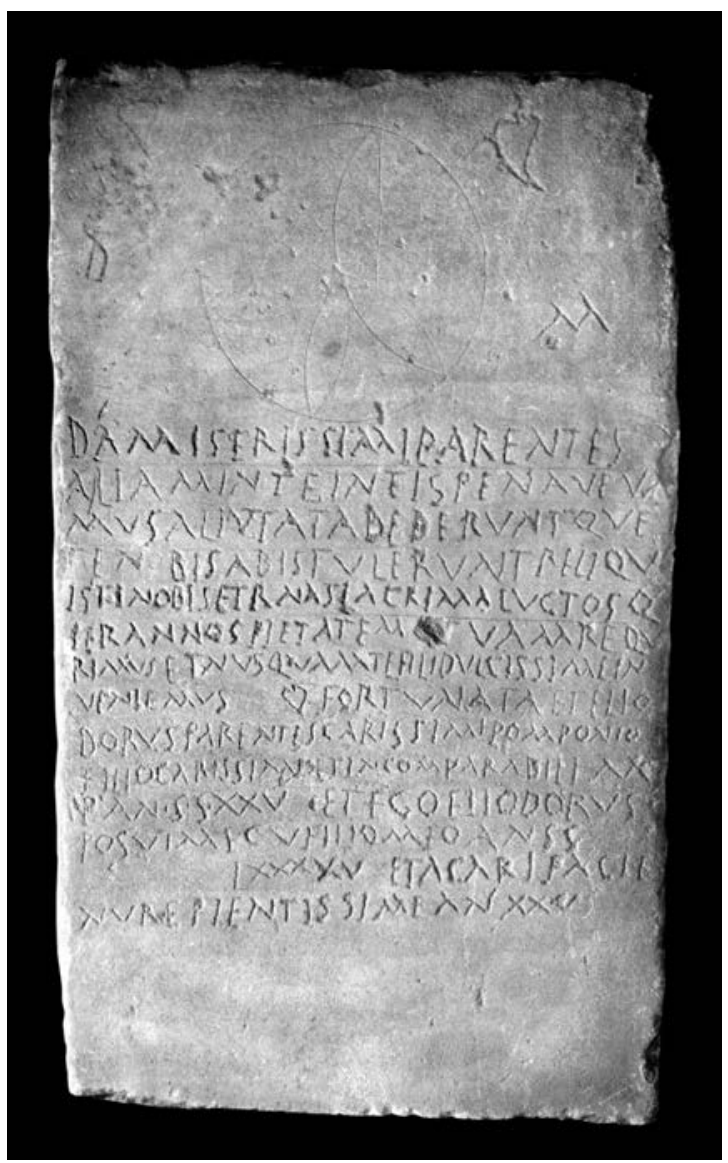

Fig. 5: Epitafio métrico de Valencia de Don Juan. Foto: cortesía de Sonia M. Martínez.

$D A$, se debe leer $D($ is $) M($ anibus $)$, aunque de la $M$ sólo se escribió la mitad en forma de una $A$ sin travesaño horizontal (fig. 5). Pero no hay que olvidar que el primer renglón ya contenía también la fórmula $D$ (is) M(anibus). Las dos primeras líneas dicen:

\section{$D($ is) M(anibus) \\ $D($ is $)\lceil M($ anibus $)\urcorner$ miserissimi parentes}

Bib.: Tovar y Bejarano 1951-1952: 21-24 (HAE 491); Rubio y Bejarano 1955: 101, n. ${ }^{\circ} 340$; Mariner 1955: 239243 (AE 1957, 37); Rodríguez 1958: 309-310; Mariner 1960: 321-322; Vives 1967: 145-147 (ILER 5811); Cugusi 1982: 103; Diego 1986: 186-188 n. . 243; Francia 1988: 13; Thigpen 1995: 20; Hernández 2001: 8-10, 76, 77, 170; Rabanal y García 2001: 271-272 n. ${ }^{\circ} 222$; Del Hoyo

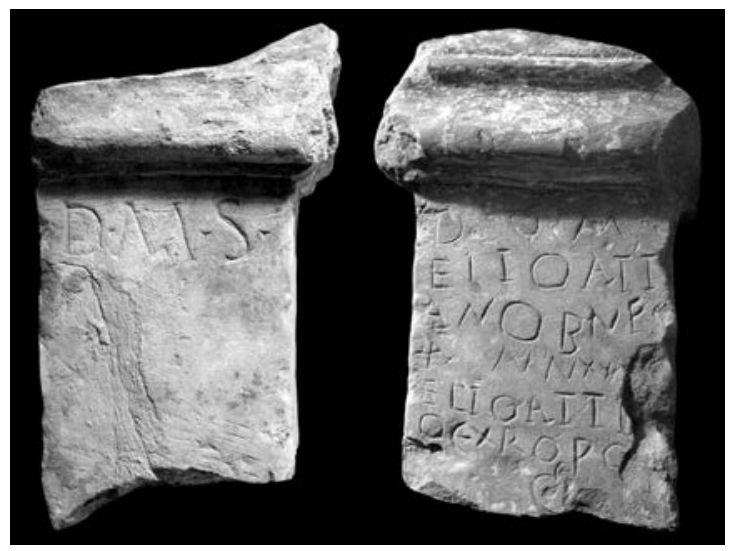

Fig. 6: Altar de Pamplona. Fotos: cortesía de J. Velaza.

2001: 27-42 (HEp 11, 2001, 301); Cugusi 2012: n. 153. - Cf. Gil 1976: 559; Alonso 1981: 82-83; Gómez Pallarès 1995: 75; Mañanes 1997: vol. I, 238; Millán 1998: 105107; Del Hoyo 2002: 142.

Según sus primeros editores, el tipo de letra del texto no es anterior al siglo III. Pero la fórmula sin el verbo no debería ser posterior a mediados o a la segunda mitad del siglo II, algo que corrobora el tipo de letra, que aconseja una datación en las décadas finales de esa centuria.

\section{PAMPLONA (NAVARRA) (CONVENTUS CAE- SARAUGUSTANUS, HISPANIA CITERIOR)}

Altar de arenisca con pie y coronamiento diferenciados, salientes a las cuatro caras, con roturas y daños múltiples en ángulos y aristas. En su cara lateral presenta únicamente una invocación a los dioses Manes, mientras que en la frontal se repite esta fórmula y sigue el texto funerario. Fue descubierto, partido en dos fragmentos, en las excavaciones de la calle de La Merced, en Pamplona.

En el lado izquierdo del altar se lee lo siguiente (fig. 6):

\section{$D($ is) M(anibus) s(acrum)}

La primera línea del texto frontal dice:

$D$ (is) M(anibus)

Bib.: Unzu y Velaza 2007: 171-173 n. 1 (AE 2008, 702; HEp 16, 2007, 484); Ollo 2008: 22; Velaza 2011: 172 y 174 (cf. AE 2011, 505); García-Barberena et al. 2014: 324-328 n. ${ }^{\circ}$. 
Los editores del monumento datan las inscripciones en el s. III (Velaza 2011: 172).

La presencia de la fórmula en la cara izquierda sólo puede justificarse como el inicio frustrado de un texto o por la presencia de líneas pintadas, como ya se ha dicho en las ediciones previas del monumento.

\section{EL SALOBRAL (ALBACETE) (CONVENTUS CAR- THAGINIENSIS, HISPANIA CITERIOR)}

Estela de arenisca con cabecera ligeramente semicircular, aparentemente cortado por la izquierda y por abajo, que presenta alisada la superficie frontal. Sus dimensiones son $(20,5)$ x (29) x 23. Las letras son muy irregulares y están mal talladas; su altura es de circ. 2,5 $\mathrm{cm}$ y las interpunciones son circulares. Fue descubierto hacia 1980 en el paraje de Los Torrejones o Los Torreones, en la finca La Florida (latitud y longitud: $38^{\circ} 51^{\prime}$ $30^{\prime \prime} \mathrm{N}, 1^{\circ} 57^{\prime} 30^{\prime \prime} \mathrm{W}$ ), a unos $3 \mathrm{~km}$ al oriente de la localidad de El Salobral. Se conserva desde agosto del año 2000 en el Museo de Albacete (inv. n. 15658), en donde lo describimos y fotografiamos el 25 de mayo de 2015 (fig. 7).

$$
\begin{array}{ll} 
& D(\text { is }) \cdot M(\text { anibus }) \cdot s(\text { acrum }) \\
& D(\text { is }) \cdot M(\text { anibus }) \cdot s(\text { acrum }) \cdot M(---) \cdot \\
& [---?] \cdot M(--)) \cdot C(---) \cdot S(---) \cdot A(--) \cdot \\
& {[---?] \cdot M(---) \cdot X X X V} \\
5 \quad & h(\text { ic }) \cdot s(\text { it- }) \cdot e(s t) \cdot \\
& -----?
\end{array}
$$

Bib.: Abascal et al. 2002: 254-255 (HEp 12, 2002, 2).

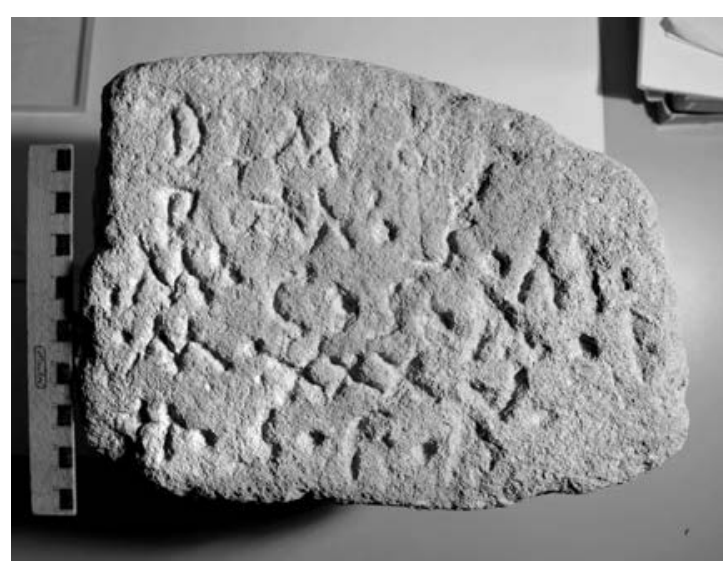

Fig. 7: Estela de El Salobral. foto: J. M. Abascal.
$D($ is) M(anibus) s(acrum) / D(is) M(anibus) s(acrum) $\{M($ arcus $)\} / M$ (arcus) C(ornelius ?) / S(- - ) alnn(orum) XXXV/ h(ic) s(itus) e(st) en Abascal et al. 2002. El tercer renglón debe contener los tria nomina del difunto, por ejemplo M(arcus) C(aecilius) o C(ornelius) S(- - -). En el tercero, la última letra puede ser la abreviatura de a(nnorum?). En el cuarto debe encontrarse la edad del difunto.

La forma de las letras y el formulario sugieren datar la inscripción en el s. III.

La repetición inicial del formulario en una estela vincula esta pieza a un epígrafe de Lugo (supra n. ${ }^{\circ} 3$ ).

\section{CASTELLAR DE SANTISTEBAN (JAÉN) (CON- VENTUS CARTHAGINIENSIS, HISPANIA CITERIOR)}

Estela de arenisca rojiza y cabecera semicircular, decorada en su parte superior con un círculo de talla muy superficial, y ligeramente dañada en su ángulo inferior derecho. Sus dimensiones son 86 x 52 x $11 \mathrm{~cm}$. El texto está grabado dentro de una tabula ansata que mide $42,5 \times 35$. La altura de las letras es de $5 \mathrm{~cm}$ en el primer renglón (situado fuera de la cartela), $6,5 / 7 \mathrm{~cm}$ en el segundo, 6 en el tercero y 8 en el cuarto. Presenta interpunciones circulares muy tenues en la primera línea. Se conserva en el Museo de Santisteban del Puerto, donde lo vimos y fotografiamos el 3 de marzo de 2017 en compañía de Joaquín Mercado Pérez, Agustín Latorre Tapia y Pilar González-Conde (fig. 8). Una copia en yeso se conserva en el Museo Arqueológico Nacional en Madrid (inv. 16697).

En la parte inferior, fuera de la cartela, junto al borde inferior de la estela y en posición invertida (fig. 9), se lee una primera fórmula $D . M$. s., seguramente correspondiente a un primer intento de talla, mientras que al comienzo del texto esta invocación aparece de nuevo. Da la impresión de que el grabador decidió empezar de nuevo tras un primer tallado defectuoso de la primera línea, para lo que se limitó a invertir la posición de la estela. Con ello no queremos descartar completamente -porque no hay argumentos contundentes en uno u otro sentido- la posibilidad de que podamos estar ante una estela reutilizada mediante el procedimiento de ponerla boca abajo y tallar la tabula ansata para suprimir el texto anterior, como ya propuso hace más de dos décadas Armin U. Stylow (1995: 225-229 n. ${ }^{\circ}$ II), en cuyo caso algunos trazos visibles al exterior de la tabula podrían ser restos del primitivo texto. 
La letra final de la primera línea es una $E$ muy estilizada que se puede confundir con una $I$ pero que en la autopsia directa del original se ve perfectamente. En la fórmula funeraria final el grabador se olvidó de insertar la $T$ correspondiente al pronombre, lo que solucionó grabando a posteriori un trazo vertical sin travesaño superior. El texto dice:

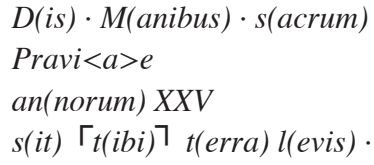

Abajo, en posición inversa:

$D($ is $) M($ anibus) s(acrum)

Bib.: CIL II 3245 a partir de datos de Manuel de Góngora y p. 949 tras haberla visto en Madrid en 1886 (de él, Vives ILER 3283, con atribución geográfica errónea); Fita 1901a: 469; Id. 1901b: 428 n. . 3, con foto (EE IX 323); Rivero 1933: 70 n. ${ }^{\circ}$ 255, a partir del vaciado de Madrid; Stylow 1995: 225-229 n. ${ }^{\circ}$ II con foto del vaciado ( $A E$ 1995, 934); González Román y Mangas 1991: 289-290 n. ${ }^{\circ}$ 256 con foto (HEp 5, 1995, 513) - Cf. Mercado Egea 1973: 55; Mercado Pérez 2015: 63-64 n. ${ }^{\circ}$ 12, con foto.

Omitimos las variantes de lectura de la bibliografía anterior a 1995, que ya fueron recogidas por Stylow en 1995 y no constituyen el objeto de estas líneas.

\section{VALORACIÓN DEL CONJUNTO}

Los ocho testimonios recogidos en las páginas precedentes no guardan ninguna relación geográfica entre sí. Sus distribución (fig. 10) muestra una dispersión arbitraria, con dos testimonios en la Baetica (n. $\left.{ }^{\circ} 1-2\right)$ y el resto en la Hispania citerior, de los cuales uno está situado en el conventus Lucensis (n. ${ }^{\circ}$ ), dos en el Asturum (n. ${ }^{\circ} 4-5$ ), uno en el Caesaraugustanus (n. ${ }^{\circ} 6$ ) y dos en el Carthaginiensis (n. ${ }^{\circ}$ 7-8).

No hay que esperar que otros testimonios que puedan aparecer desvirtúen esta impresión de que se trata de ejemplos debidos a la iniciativa individual de los artesanos que grabaron las piezas o a los accidentes técnicos ocurridos durante el proceso de tallado. No cabe duda de que ejemplos como el de la inscripción de Santibáñez de Vidriales (n. ${ }^{\circ}$ ) pueden considerarse ensayos del grabador

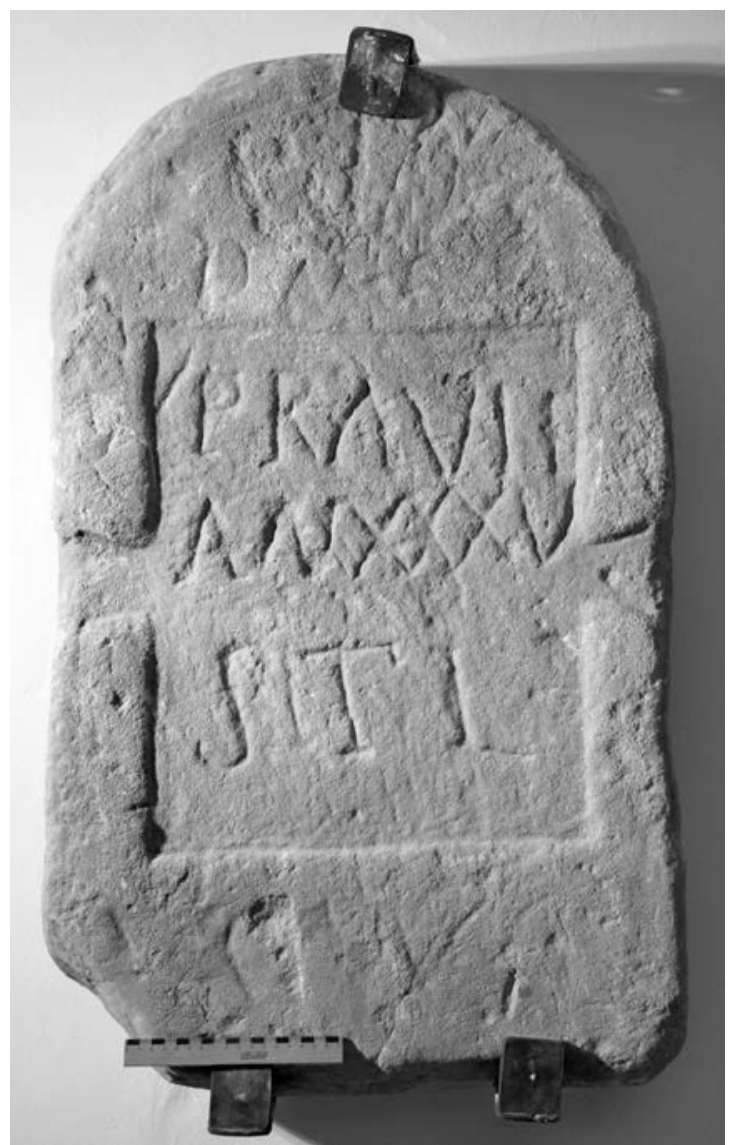

Fig. 8: Estela de Castellar de Santisteban. Foto: J. M. Abascal.

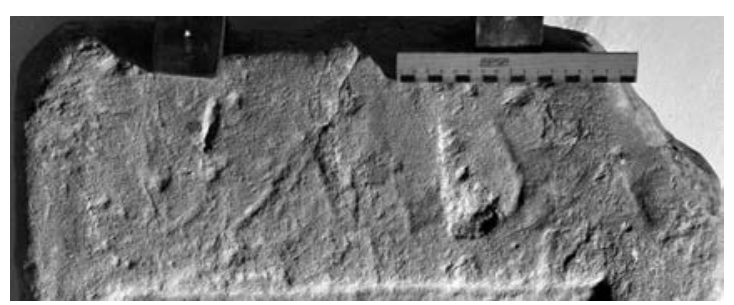

Fig. 9: Detalle de la parte inferior de la estela de Castellar de Santisteban. Foto: J. M. Abascal.

que no fueron borrados a posteriori; otros, como el de Lugo (n. ${ }^{\circ}$ ), pueden explicarse por el hecho de que los personajes fallecidos que se citan en el texto son dos; en Pamplona (n. ${ }^{\circ}$ ) podríamos esperar un texto pintado debajo de la invocación de la cara izquierda, en Castellar de Santisteban (n. ${ }^{\circ}$ 8) se reutilizó el soporte al invertirlo, etc., etc. Una casuística muy diversa permite explicar 


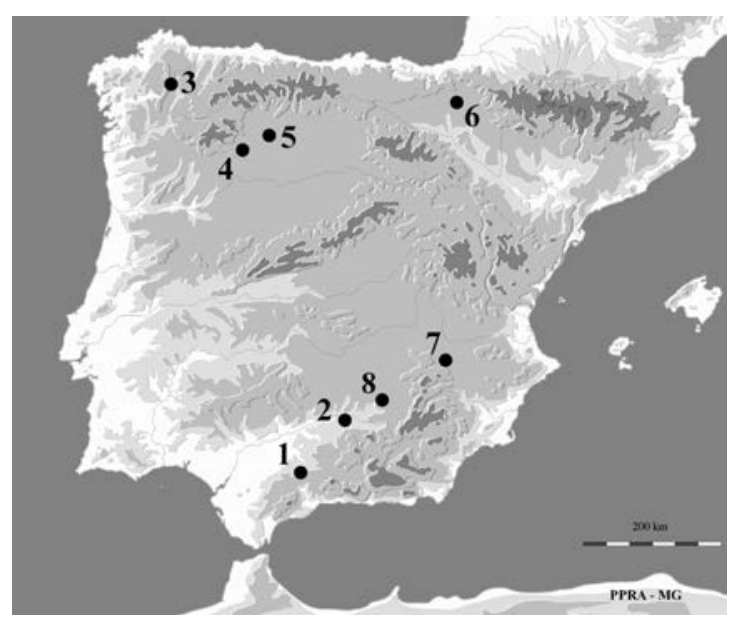

Fig. 10: Mapa de dispersión de los testimonios citados en el texto (J. M. Abascal sobre base cartográfica de P. P. Ripollès y M. Gozalbes).

casi todos los testimonios que han llegado hasta nosotros de esa repetición de la invocación a los dioses Manes, por lo que hay que descartar cualquier referencia a modas o estilos que animaran a esta práctica.

El fenómeno no se restringe exclusivamente a la península Ibérica. De hecho, la repetición de la fórmula $D$ (is) M(anibus) s(acrum) en un mismo epígrafe es un hecho recurrente, especialmente en las inscripciones del norte de África. A los ejemplos y paralelos ya citados en las páginas precedentes, hay que añadir un epígrafe de Gafsa (Africa proconsularis) en donde la invocación aparece por primera vez como encabezamiento de todo el monumento e, inmediatamente debajo, como inicio del epitafio de uno de los dos difuntos (CIL VIII, 125) y en la misma localidad se encuentra una iteración consecutiva de la fórmula en la misma línea, es decir, D.M.s.D. [M.s.] (CIL VIII, 133). En otro texto de Sidi Ali Benyoub (Mauretania Caesariensis. CIL VIII 21717) las dos primeras líneas del texto dicen $D($ is) $M$ (anibus) s(acrum) / $D($ is $)$ s(acrum). Un altar de Aubord (Gallia Narbonensis) conservado en el Museo de Nîmes (ILGN 525; Provost 1999: 170) presenta en un lado los bustos de sendos difuntos con la invocación $D$ (is) M(anibus) s(acrum), mientras que en el lado opuesto se encuentra el texto funerario completo precedido de nuevo por la invocación $D($ is $)$ M(anibus) s(acrum).

Un altar funerario de Roma (CIL VI 33971) presenta también la doble invocación $D$. M. al comienzo del texto. $\mathrm{Y}$ en otro de la misma procedencia (CIL VI 18422), la primera aparición de esta fórmula se localiza dentro de una corona en el frontón del monumento, mientras que la segunda da inicio al texto en una cartela de la parte media.

No entramos a valorar los numerosos ejemplos de textos con doble o triple columna, encabezada en cada caso por una invocación, que testimonian el enterramiento bajo un solo monumento de dos o más difuntos. Esta práctica, muy extendida entre las estelas de la mitad $\mathrm{N}$ de Hispania, fundamentalmente en el área del Duero (CIL II 867, 2627, AE 1983, 508, AE 2000, 765, AE 2009, 551; HEp 2003/04, 555 у 573; HEp 7, 1997, 652 у 654, etc.), es corriente en otras provincias romanas. Únicamente habría que destacar, especialmente en la provincia de Africa proconsularis, la existencia de monumentos que presentan la invocación D. M. s. en una columna y el texto funerario con una nueva invocación en la columna contigua, como si se tratara de monumentos sin terminar (Gsell 1922: n. $786,1787,2509$; además, CIL VIII 549, 565 y 568 , los tres de Thala); incluso conocemos testimonios de esa doble columna con sendas fórmulas D. M. s. pero sin texto en la parte inferior (Gsell 1922: n. ${ }^{\circ} 122$ [CIL VIII 5193]).

En cuanto a la cronología, dado que las repeticiones formulares que hemos presentado lo son de expresiones que se generalizan en los ss. II y III, huelga decir que estamos ante un fenómeno posterior a época flavia.

\section{NOTA}

1. Este trabajo se ha realizado en el marco del proyecto de investigación Sociedad romana y hábito epigráfico en la Hispania citerior, HAR2015-65168-P (MINECO/FEDER), subvencionado por el Ministerio de Economía y Competitividad del Gobierno de España.

\section{BIBLIOGRAFÍA}

ABASCAL, J. M. (2016): Officina epigráfica de Sansueña (Rosinos y Santibáñez de Vidriales, Zamora), A Baete ad fluvium Anam: Cultura epigráfica en la Bética occidental y territorios fronterizos. Homenaje al profesor José Luis Moralejo Álvarez (J. Carbonell, H. Gimeno, eds.), Alcalá de Henares, 345-360.

ABASCAL, J. M.; ZARZALEJOS, M. M.; SANZ, R. (2002): Los Torreones (El Salobral, Albacete): nuevos documentos de ocupación romana, Segundo Congreso de Historia de Albacete (22 al 25 de noviembre de 2000), vol. I. Arqueología y Prehistoria, Albacete, 253-269. 
ABÁSOLO, J. A.; GARCÍA ROZAS, R. (1990): Sobre las estelas zamoranas y su ornamentación, Primer Congreso de Historia de Zamora. Tomo 2: Prehistoria y mundo antiguo, Zamora, 545-560.

ALONSO, J. L. (1981): Historia antigua y medieval de la comarca de Los Oteros, León.

ALONSO ÁVILA, Á.; CRESPO, S. (2000): Corpus de inscripciones romanas de la provincia de Zamora. Fuentes epigráficas para la historia social de la Hispania romana, Valladolid.

ARIAS, F.; LE ROUX, P.; TRANOY, A. (1979): Inscriptions romaines de la province de Lugo, Paris.

ATENCIA, R. (1971): Inscripciones latinas funerarias en la alcazaba de Málaga, Málaga 11, 1971,31-40.

BLÁZQUEZ, Á. (1923): Inscripción inédita de Santibáñez de Vidriales, Boletín de la Real Academia de la Historia, 82, 333.

BRAGADO, J. M. (1990): Fuentes literarias y epigráficas de la provincia de Zamora y su relación con las vías romanas de la cuenca del Duero (Tesis doctoral en microficha), León.

CORELL, J.; GÓMEZ, X. (2002-2003): Dues inscripcions de la provincia de Jaén, Studia Philologica Valentina 6, 239-246.

CUGUSI, P. (1982): Carmina Latina Epigraphica e tradizione letteraria, Epigraphica 44, 65-107.

CUGUSI, P. (2012): Carmina Latina Epigraphica Hispanica post Buechelerianam collectionem editam reperta cognita, Faenza.

DIEGO, F. (1986): Inscripciones romanas de la provincia de León, León.

D’ORS, A.; VÁZQUEZ, M. (1949): Nueva lápida romana, Boletín de la Comisión Provincial de Monumentos Históricos y Artísticos de Lugo 3, n. ${ }^{\circ}$ 29-30, 205-207.

FITA, F. (1901a): Epigrafía romana de Montánchez, Rena, Baños de la Encina, Linares, Santiesteban del Puerto, Cartagena y Cádiz, Boletín de la Real Academia de la Historia 38, 450-473.

FITA, F. (1901b): Nuevas inscripciones romanas de la provincia de Jaén, Boletín de la Real Academia de la Historia 39, 420-429.

FRANCIA, R. (1988): Notas de antroponimia al Thesaurus Linguae Latinae, Analecta Malacitana 11.1, 11-24.

GARCÍA-BARBERENA, M.; UNZU, M.; VELAZA, J. (2014): Nuevas inscripciones romanas de Pompelo, Epigraphica 76, 2014, 323-344

GIL, J. (1976): Epigraphica, Cuadernos de Filología Clásica 11, 1976, 545-574.

GÓMEZ PALLARÈS, J. (1995): Initia de los Carmina Latina Epigraphica Hispaniae (Conventus Tarraconensis, I), Faventia 17.1,67-86.

GÓMEZ VILA, J. (2009): Epigrafía romana de la provincia de Lugo, London.

GONZÁLEZ ROMÁN, C.; MANGAS, J. (1991): Corpus de inscripciones latinas de Andalucía. Vol. III: Jaén, Sevilla, 2 vol.

GOZALBES, C.; MUÑOZ, F. (1986): Puente Piedra: la vía romana de la sal, Jábega 53, 1986, 20-23.
GSELL, St. (1922): Inscriptions latines de l'Algérie. Tome premier. Inscriptions de la proconsulaire. Paris.

HERNÁNDEZ GUERRA, L. (1999): Epigrafía romana de unidades militares relacionadas con Petavonium. Rosinos de Vidriales (Zamora). Estudio social, religioso y prosopográfico, Valladolid.

HERNÁNDEZ PÉREZ, R. (2001): Poesía latina sepulcral de la Hispania Romana: estudio e los tópicos y sus formulaciones, Cuadernos de Filología. Anejo LXIII, València.

DEL HOYO, J. (2001): Nuevo estudio del carmen epigraphicum de Pomponio, Studia Philologica Valentina 5, 27-42.

DEL HOYO, J. (2002): La ordinatio en los CLE Hispaniae, en, Asta ac pellege. 50 años de la publicación de Inscripciones hispanas en verso de S. Mariner (J. del Hoyo; J. Gómez Pallarès, eds.), Madrid 2002, 143-162.

ILER vide VIVES ILER.

$I L G N=$ Inscriptions latines de Gaule Narbonnaise.

LIÓN, M. C. (1990): Aspectos decorativos y onomásticos de las estelas funerarias del occidente de Zamora, Primer Congreso de Historia de Zamora. Tomo 2: Prehistoria y mundo antiguo, Zamora, 561-569.

MAÑANES, T. (1997): Roma en León, Historia de León, Tomo I. Prehistoria, Edad Antigua, Edad Media, León, 157-352.

MARINER, S. (1955): Nuevas inscripciones latinas de España, con introducción acerca de los recientes hallazgos epigráficos barceloneses por Durán y Sampere, AEA 28, 197-243.

MARINER, S. (1960): Loci similes virgilianos en epígrafes hispánicos de reciente aparición, Emerita 28, 317-326.

MARTÍN VALLS, R. (1975): Epígrafes romanos de Sansueña (Rosinos y Santibáñez de Vidriales), Sobre los campamentos de Petavonium (R. Martín, G. Delibes, eds.), Santiago de Compostela, 13-18.

MERCADO EGEA, J. (1973): La muy ilustre villa de Santisteban del Puerto, Madrid.

MERCADO PÉREZ, J. (2015): Epigrafía latina del municipio romano de Ilugo en el Alto Guadalquivir, en Reino de Jaén, Crónica Digital de Investigación Local de la Provincia 5, 47-68.

MILLÁN, M. A. (1998): Historia de Coyanza, Valencia de Don Juan.

MORÁN, C. (1937): Neue lateinische Inschriften aus Spanien, Sitzungsberichte der Preussischen Akademie der Wissenschaften 1937 (18), 142-149.

OLLO, M. (2008): Pamplona romana, La Pamplona reencontrada (J. L. Ramírez Sádaba, ed.). Pamplona.

PASTOR, M.; RODRÍGUEZ MARTÍN, J. A. (1999-2000): Inscripciones romanas inéditas de Alameda, Archidona, Mollina (Málaga) y Los Corrales (Sevilla), Mainake 21-22, 217-234.

PASTOR, M.; STYLOW, A. U. (1996): Miscelánea epigráfica de la provincia de Jaén II/III, Anales de Arqueología Cordobesa 7, 283-292.

PROVOST, M. et al. (1999): Carte archéologique de la Gaule 30/2. Le Gard. Paris. 
RABANAL, M. A.; GARCÍA, S. M. (2001): Epigrafía romana de la provincia de León: revisión y actualización, León.

RIVERO, C. M.del (1933): El lapidario del Museo Arqueológico Nacional., Catálogo ilustrado de las inscripciones latinas, Madrid.

RODRÍGUEZ, J. (1958): Nuevas inscripciones sepulcrales, Archivos Leoneses 12, 309-314.

RODRÍGUEZ COLMENERO, A. (2011): Lucus Augusti. La ciudad romano-germánica del Finisterre ibérico. Génesis y evolución histórica (14 a.C. - 711 d.C.), Lugo

RODRÍGUEZ LAGE, S. (1974): Las estelas funerarias de Galicia en la época romana, Orense.

RUBIO, L.; BEJARANO, V. (1955): Documenta ad linguae Latinae historiam ilustrandam, Madrid.

SERRANO, E.; ATENCIA, R. (1981): Inscripciones latinas del Museo de Málaga, Madrid.

STYLOW, A. U. (1995): Miscelánea epigráfica de la provincia de Jaén IV. Problemas de datación de las inscripciones tardías, Anales de Arqueología Cordobesa 6, 217-237.

THIGPEN, J. B. (1995): A Literary Analysis of Latin Epitaphs from Roman Spain which contain creative Biographical Discourse, Diss., Chapel Hill.
TOVAR, A.; BEJARANO, V. (1951-1952): Una inscripción latinovulgar de Valencia de Don Juan, Boletín del Seminario de Estudios de Arte y Arqueología 18, 21-24.

UNZU URMENETA, M.; VELAZA, J. (2007): Hallazgos de la calle La Merced y asociados (Pamplona) en La tierra te sea leve. Arqueología de la muerte en Navarra, Pamplona, 169-175.

VÁZQUEZ SACO, F.; VÁZQUEZ SEIJAS, M. (1954): Inscripciones romanas de Galicia II. Provincia de Lugo, Santiago de Compostela.

VÁZQUEZ SEIJAS, M. (1948-1949): Museo Provincial de Lugo, Memorias de los Museos Arqueológicos Provinciales 9-10, 183-187.

VELAZA, J. (2011): Crónica de epigrafía antigua de Navarra III, VII Congreso General de Historia de Navarra: Arqueología. Historia Antigua. Historia Medieval. Historia del Arte y de la Música, volumen I, Pamplona, 169-176.

VIVES, J. (1967): Inscripciones leonesas de época romana, Archivos Leoneses 41, 145-147.

VIVES, J. (1971-1972): Inscripciones latinas de la España romana. Antología de 6.800 textos I-II, Barcelona. 